\title{
The treatment choices and outcome of hepatocellular carcinoma in hemophilic patients with human immunodeficiency virus/ hepatitis C virus (HIV/HCV) coinfection due to contaminated blood products in Japan
}

\author{
Mitsuhisa Takatsuki ${ }^{1} \wedge$, Koji Natsuda ${ }^{2} \wedge$, Masaaki Hidaka ${ }^{2} \wedge$, Koji Sawada ${ }^{3} \wedge$, Motohiro Shindo $^{3} \wedge$, \\ Tomoyuki Endo $^{4} \wedge$, Takeshi Hagiwara ${ }^{5} \wedge$, Hiroshi Yotsuyanagi ${ }^{6} \wedge$, Tomohiko Koibuchi ${ }^{6} \wedge$, \\ Kunihisa Tsukada $^{7} \wedge$, Haruka Uemura ${ }^{7} \wedge$, Kazuhiko Hayashi ${ }^{8} \wedge$, Tomoko Uehira ${ }^{9}$, Eiji Mita ${ }^{10}$, \\ Masahiro Yamamoto ${ }^{11}$, Soichiro Takahama ${ }^{11}$, Susumu Eguchi ${ }^{2} \wedge$
}

${ }^{1}$ Department of Digestive and General Surgery, Graduate School of Medicine, University of the Ryukyus, Okinawa, Japan; ${ }^{2}$ Department of Surgery, Graduate School of Biomedical Sciences, Nagasaki University, Nagasaki, Japan; ${ }^{3}$ Division of Gastroenterology and Hematology/Oncology, Department of Medicine, Asahikawa Medical University, Asahikawa, Japan; ${ }^{4}$ Department of Hematology, Hokkaido University Hospital, Sapporo, Japan; ${ }^{5}$ Department of Laboratory Medicine, Tokyo Medical University, Tokyo, Japan; ${ }^{6}$ Division of Infectious Diseases, Advanced Clinical Research Center, Institute of Medical Science, The University of Tokyo, Tokyo, Japan; ${ }^{7}$ AIDS Clinical Center, National Center for Global Health and Medicine, Tokyo, Japan; ${ }^{8}$ Department of Gastroenterology and Hepatology, Federation of National Public Service Personnel Mutual Aid Associations, Meijo Hospital, Nagoya, Japan; ${ }^{9}$ AIDS Medical Center, National Hospital Organization Osaka National Hospital, Osaka, Japan; ${ }^{10}$ Department of Gastroenterology and Hepatology, National Hospital Organization Osaka National Hospital, Osaka, Japan; ${ }^{11}$ National Hospital Organization, Kyushu Medical Center, AIDS/HIV Combined Clinic Center, Fukuoka, Japan

Contributions: (I) Conception and design: M Takatsuki, K Natsuda; (II) Administrative support: S Eguchi; (III) Provision of study materials or patients: M Takatsuki, K Natsuda, M Hidaka; (IV) Collection and assembly of data: M Hidaka, K Sawada, M Shindo, T Endo, T Hagiwara, H Yotsuyanagi, T Koibuchi, K Tsukada, H Uemura, K Hayashi, T Uehira, E Mita, M Yamamoto, S Takahama; (V) Data analysis and interpretation: M Takatsuki, K Natsuda, M Hidaka; (VI) Manuscript writing: All authors; (VII) Final approval of manuscript: All authors.

Correspondence to: Dr. Mitsuhisa Takatsuki. Department of Digestive and General Surgery, Graduate School of Medicine, University of the Ryukyus, Okinawa 903-0215, Japan. Email: mtaka1@med.u-ryukyu.ac.jp.

\begin{abstract}
Background: Human immunodeficiency virus (HIV) and hepatitis C virus (HCV) coinfection through unheated blood product for hemophilia caused in early 1980s has been significantly serious problem in Japan. After the development of HIV treatment in 1990s, HCV-related hepatocellular carcinoma (HCC) has been one of the most significant problem in these population. Treatment choices for HCC might be limited in hemophilia patients because of their bleeding tendency. The aim of this study was to elucidate the treatment choices and outcome of HCC in hemophilic patients coinfected with HIV/HCV due to contaminated blood products.
\end{abstract}

Methods: We asked 444 Japanese centers that specialize in treating HIV patients for participation, whether they have HIV/HCV coinfected cases with HCC, and the patient characteristics, treatments for HCC and survival after treatments were retrospectively reviewed according to each institutional medical records.

Results: Of 444 centers, 139 centers (31\%) responded to the first query, and 8 centers (1.8\%) ultimately provided 26 cases of HCC in coinfected hemophilic patients, diagnosed between December 1999 and

\footnotetext{
^ ORCID: Mitsuhisa Takatsuki, 0000-0002-0819-3156; Koji Natsuda, 0000-0003-1506-5627; Masaaki Hidaka, 0000-0001-8191-6405; Koji Sawada, 0000-0002-9708-4786; Motohiro Shindo, 0000-0003-0754-9568; Tomoyuki Endo, 0000-0002-2800-2492; Takeshi Hagiwara, 0000-0002-3337-4450; Hiroshi Yotsuyanagi, 0000-0001-7882-5262; Tomohiko Koibuchi, 0000-0001-7736-6922; Kunihisa Tsukada, 00000002-2524-8884; Haruka Uemura, 0000-0002-2096-8620; Kazuhiko Hayashi, 0000-0002-7919-6071; Tomoko Uehira, 0000-0001-71809809; Masahiro Yamamoto, 0000-0003-0785-3607; Susumu Eguchi, 0000-0002-7876-0152.
} 
December 2017. All 26 were male hemophilic patients, with a median age at HCC diagnosis of 49 (range, 34-73) years. Thirteen cases (50\%) were HCV-RNA positive, and 14 cases (54\%) had a solitary tumor. Even in the cases of Child-Pugh grade A, only 1 case underwent resection, and 18 cases (69\%) did not receive the standard treatment recommended by the Japanese Society of Hepatology.

Conclusions: Hemophilic HCC patients with HIV/HCV coinfection may not routinely receive standard treatment due to their bleeding tendency and several complications related to HIV/HCV coinfection.

Keywords: Human immunodeficiency virus (HIV); hepatitis C virus (HCV); coinfection; hemophilia; hepatocellular carcinoma (HCC)

Submitted Mar 24, 2021. Accepted for publication Oct 25, 2021.

doi: 10.21037/jgo-21-157

View this article at: https://dx.doi.org/10.21037/jgo-21-157

\section{Introduction}

In the early 1980s, unheated contaminated blood products for hemophilia caused a crisis of human immunodeficiency virus (HIV)/hepatitis C virus (HCV) coinfection in Japan, so that it was considered as extremely serious social problem, and many patients have been suffered from this irrational iatrogenic disease for years $(1,2)$.

After the introduction of antiretroviral therapy (ART) for HIV in the mid-1990s, acquired immunodeficiency syndrome (AIDS) was replaced by HCV-related liver disease as the main cause of death in these patients $(1,2)$. Hepatocellular carcinoma (HCC) is well recognized as the fatal complication of HCV cirrhosis, and its treatment has been standardized both in Western countries (3) and in Japan (4).

Generally, standard treatment for HCC might be considered riskier in HIV/HCV coinfected patients, especially hemophilic cases, due to the concern of bleeding. As a result, outcomes for HCC in this population have been reported to be generally poor $(5,6)$. Although surgery, including liver transplantation (LT) $(7,8)$, has been reported as feasible in hemophilic patients, physicians might hesitate to refer such patients not only to surgery, but also to less invasive procedures such as radio frequency ablation (RFA).

Because there have been no data on the characteristics of HCC in hemophilic patients with HIV/HCV coinfection due to contaminated blood products in Japan, we performed a nationwide survey asking centers to elucidate treatments and outcomes in such patients.

The aim of this study was to elucidate the characteristics of HCC in hemophilic patients with HIV/HCV coinfection, based on the data accumulated from multicenter in Japan.

We present the following article in accordance with the STROBE reporting checklist (available at https://dx.doi. org/10.21037/jgo-21-157).

\section{Methods}

\section{Study design and patients}

We asked Japanese centers that specialize in treating HIV patients for participation, whether they have HIV/HCV coinfected cases with HCC, and the patient characteristics, treatments for HCC and survival after treatments were retrospectively reviewed according to each institutional medical records. The definition of HIV/HCC co-infection was positive of antibody both for HIV and HCV. The diagnosis of HCC was made according to each institutional decision, based on typical imaging findings, biopsy, or surgical specimen in the case with hepatectomy or LT. Accordingly, no definitive diagnosis might be made for the cases without pathological specimen. This study was carried out as the part of the research project of HIV/ $\mathrm{HCV}$ coinfection due to blood products in Japan ("Eguchi project") and approved first by the institutional review board of Nagasaki University Hospital (approval No. 16062709), and then by the review boards of all participating institutions. In principle, written informed consent was not necessary for retrospective and observational study depending on each institutional regulation, but instead of it, the contents of the study were informed on website in Japanese (https://www.med.nagasaki-u.ac.jp/surgery2/hlw_ kaken/index.html). The study was conducted in accordance with the Declaration of Helsinki (as revised in 2013).

\section{Statistical analysis}

To clarify the patient characteristics in this retrospective 
Table 1 Characteristics of the patients at HCC diagnosis $(n=26)$

\begin{tabular}{lc}
\hline Characteristics & Value \\
\hline Gender & 26 \\
Male & \\
Age & $49(34-73)$
\end{tabular}

Hemophilia type

A

B

Child-Pugh score

Child-Pugh grade

$\begin{array}{ll}\text { A } & 17 \\ \text { B } & 7 \\ \text { C } & 2\end{array}$

HIV treatment

Yes

No

20

6

HIV-RNA

Positive

10

Undetectable

16

CD4 count

379 (25-815)

HCV treatment

Yes

No

11 (3 with DAA)

15

HCV-RNA

Positive

Undetectable

Unknown

HCC size (mm)

HCC nodule (number)

HCC solitary or multiple

$\begin{array}{lc}\text { Solitary } & 14 \\ \text { Multiple } & 12 \\ \text { AFP }(\mathrm{ng} / \mathrm{mL}) & 19.2(2.7-13,095) \\ \text { PIVKA-II }(\mathrm{mAU} / \mathrm{mL}) & 32.0(8.0-44.000) \\ \text { HCC primary treatment } & 22 \\ \text { Yes } & 13 \\ \text { TACE } & \end{array}$

Table 1 (continued)
Table 1 (continued)

\begin{tabular}{lc}
\hline Characteristics & Value \\
\hline RFA & 7 \\
TACE + RFA & 1 \\
Resection & 1 \\
No & 4
\end{tabular}

Data are presented as number or median (range). HCC, hepatocellular carcinoma; HIV, human immunodeficiency virus; $\mathrm{HCV}$, hepatitis $\mathrm{C}$ virus; DAA, direct acting antivirals; AFP, alfa fetoprotein; PIVKA-II, protein induced by vitamin $\mathrm{K}$ absence or antagonist-II; TACE, transcatheter arterial chemoembolization; $\mathrm{RFA}$, radiofrequency ablation.

study, the pre- and post-treatment parameters examined included the gender, age, hemophilia type, Child-Pugh grade, score, HIV treatment, HIV-RNA, CD4 count, HCV treatment, HCV-RNA, HCC size, number of the HCC nodules, range of solitary tumor (\%), tumor marker, primary treatment. Continuous variables are described as median (range from minimum to maximum), categorical variables are described as percentages. We described these parameters in Tables 1,2 in detail.

The survival was analyzed from the day of treatment to the most recent follow-up. The overall survival rates were assessed with the Kaplan-Meier method using the logrank test. Differences were considered to be statistically significant for value of $\mathrm{P}<0.05$. Statistical analyses were performed using the SAS ver. 9.4 software (SAS, Cary, NC, USA).

\section{Results}

\section{The characteristics of the patients}

We firstly asked 444 Japanese centers that specialize in treating HIV patients for participation, whether they have HIV/HCV coinfected cases with HCC. Of them, 139 centers $(31 \%)$ responded to the first query, and 8 centers $(1.8 \%)$ ultimately provided 26 cases of HCC in coinfected hemophilic patients, diagnosed between December 1999 and December 2017. All patients were certified as the victim of the infection due to contaminated or unheated blood products for hemophilia, which were imported to Japan in early 1980s (1,2). The characteristics of the 26 subjects are listed in Table 1.

The median age of the patients at HCC diagnosis was 
Table 2 HCC treatment according to the Child-Pugh grade

\begin{tabular}{|c|c|c|c|}
\hline Characteristics & Child-Pugh A $(n=17)$ & Child-Pugh B $(n=7)$ & Child-Pugh C $(n=2)$ \\
\hline Age at diagnosis & $53(44-73)$ & $45(37-65)$ & 34,36 \\
\hline Size $(\mathrm{mm})$ & $21(7-80)$ & $21(13-100)$ & 20,10 \\
\hline Solitary/multiple & $9 / 8$ & $4 / 3$ & $1 / 1$ \\
\hline \multicolumn{4}{|l|}{ Primary treatment } \\
\hline TACE & 10 & 3 & 0 \\
\hline RFA & 5 & 2 & 0 \\
\hline TACE + RFA & 1 & 0 & 0 \\
\hline Resection & 1 & 0 & 0 \\
\hline None & 0 & 2 & 2 \\
\hline JSH guideline met/unmet & $7 / 10$ & $1 / 6$ & $0 / 2$ \\
\hline EASL guideline met/unmet & $9 / 8$ & $4 / 3$ & $0 / 2$ \\
\hline \multicolumn{4}{|l|}{ Outcome } \\
\hline Alive without HCC & 2 & 1 & 0 \\
\hline Alive with HCC & 5 & 1 & 0 \\
\hline Died without HCC & 0 & 0 & 0 \\
\hline Died with HCC & 9 & 5 & 2 \\
\hline Unknown & 1 & 0 & 0 \\
\hline
\end{tabular}

Data are presented as number or median (range). HCC, hepatocellular carcinoma; TACE, transcatheter arterial chemoembolization; RFA, radiofrequency ablation; JSH, Japanese Society of Hepatology; EASL, European Association for the Study of the Liver.

49 (range, 34-73) years. The date of HCC diagnosis ranged from June 1999 to December 2015. All cases were positive for HCV antibody, and the majority had compensated cirrhosis as defined by Child-Pugh A (65\%). HIV-RNA and HCV-RNA at diagnosis of HCC were positive in 10 (38\%) and 13 cases (50\%), respectively. Of the $13 \mathrm{HCV}$-RNA positive cases, only 4 cases had received HCV treatment with interferon (IFN) before HCC diagnosis; among them was 1 case with and 3 without ribavirin treatment. Of the $9 \mathrm{HCV}-\mathrm{RNA}$ negative cases, 6 had received $\mathrm{HCV}$ treatment [IFN with ribavirin in 3 and direct-acting antivirals (DAA) in 3 (2 with sofosbuvir and 1 with simeprevir)], while the other 3 had no history of HCV treatment. The median size of HCC lesions was $21 \mathrm{~mm}$, and 14 cases (54\%) had only a solitary tumor. Twenty-two cases $(85 \%)$ underwent primary treatment for HCC, mainly transcatheter arterial chemoembolization (TACE) in 14 cases $(54 \%)$. Only 1 case underwent hepatectomy. In one case, LT was finally performed for recurrent HCC with decompensated cirrhosis after unsuccessful RFA and subsequent repeated TACE.

\section{Treatment for HCC according to Child Pugh grade}

Primary treatments for HCC and outcomes according to Child-Pugh grade are shown in Table 2. Even among Child-Pugh A patients, the most frequent treatment was TACE; only 1 case underwent hepatectomy for small solitary HCC $(17 \mathrm{~mm})$. As mentioned before, 1 case that underwent RFA finally received a deceased donor LT after repeated TACE for recurrent HCC. Although the date of HCC diagnosis ranged from 1999 to 2015, for consistency's sake we used current Japanese (4) and European (3) guidelines to check whether patients had received recommended treatment. Given a general assumption of good performance status, the cases which met the recommended treatments in the current guideline of Japan Society of Hepatology (JSH) (4) and European Association for the Study of the Liver (EASL) (3) were 7 (41\%) and 9 (53\%) in Child-Pugh A, 1 (14\%) and 4 (57\%) in Child-Pugh B, and 0 for both in Child-Pugh C (LT was recommended in both JSH and EASL guidelines), respectively. 

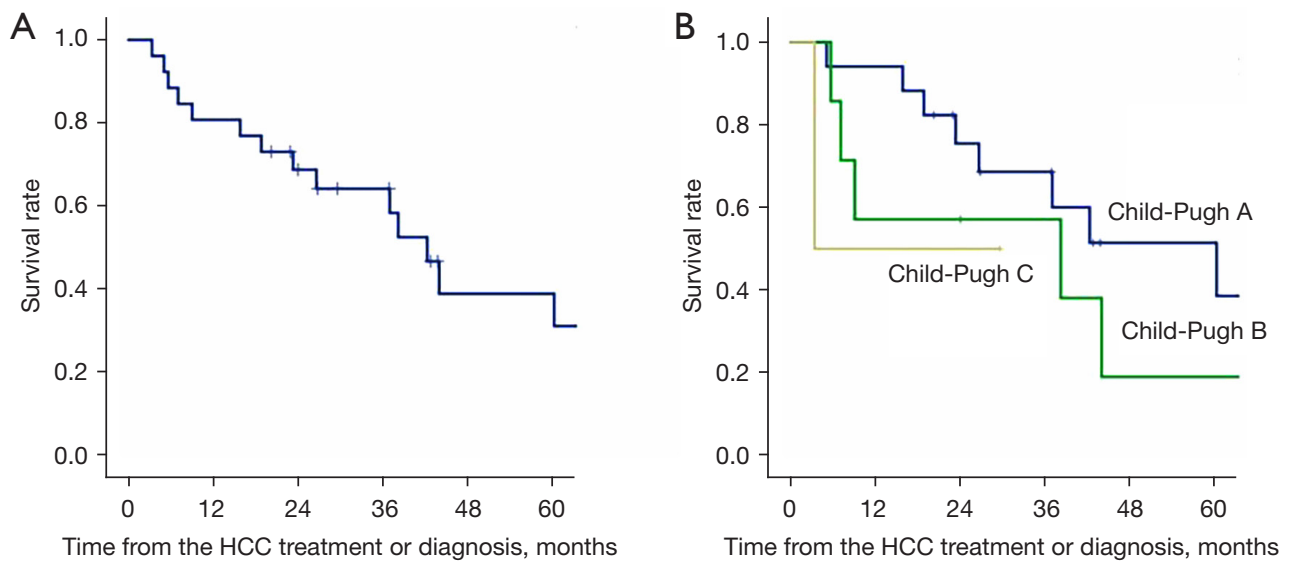

Figure 1 Overall survival (A) and patient survival according to the Child-Pugh grade (B) after the initial treatment or diagnosis of HCC in HIV/HCV coinfection. Child-Pugh A (n=17), B (n=7) and C (n=2). Four patients (2 in Child-Pugh B and 2 in C) did not receive any treatments for HCC, so that survival in these patients were from the time of HCC diagnosis. HCC, hepatocellular carcinoma; HIV, human immunodeficiency virus; $\mathrm{HCV}$, hepatitis $\mathrm{C}$ virus.

\section{Overall survival according to the Child-Pugh grade}

The patient survival after the diagnosis or the first treatment of HCC is shown in Figure 1. Overall survival rates at 1, 3 and 5 years after primary treatment or diagnosis for HCC were $80.8 \%, 64.2 \%$ and $38.9 \%$, respectively.

In the 14 Child-Pugh A cases with lesion size and number less than or equal to $3 \mathrm{~cm}$ and 3, respectively, the 1-, 3 - and 5 -year survival rates were $92.9 \%, 84.4 \%$ and $63.3 \%$.

\section{Discussion}

This is the report of the characteristics of HCC from the point of view of the treatment choices and the outcome, in $\mathrm{HIV} / \mathrm{HCV}$ coinfection due to contaminated blood products for hemophilia in Japan. Eight centers provided data from 26 cases, and our analysis revealed that the majority did not receive the standard treatments proposed in the guidelines, either from Japan (4) or Europe (3). Even among the ChildPugh A cases, only 1 patient underwent hepatectomy, and more than half received TACE only, possibly due to concerns about bleeding even with RFA. As a result, overall survivals in these population were tended to be worse than the reported outcomes in the updated HCC registry of the Liver Cancer Study Group of Japan (LCSGJ) (9): $83.4 \%, 63.4 \%$ and $48.4 \%$, despite the much younger age of patients in our HIV/HCV coinfection cohort (median age: 49 years) compared to patients in the LCSGJ registry (median age: 69.5 years) (9). Because the 1980s crisis of contaminated blood products mostly affected small children with hemophilia, the age at diagnosis of HCC in HIV/ $\mathrm{HCV}$ coinfected patients in this study was much younger than that of HCC patients registered in LCSGJ, by roughly 20 years. Thus, the actual outcome of the patients can be considered significantly worse in HIV/HCV coinfection, taking into account the age of the patients. Even in the Child-Pugh A cases with lesion size and number less than or equal to $3 \mathrm{~cm}$ and 3 , the 1-, 3- and 5-year survival rates were $92.9 \%, 84.4 \%$ and $63.3 \%$, which were worse than the values of Child-Pugh A cases in the LCSGJ registry that underwent hepatectomy for the same size and number: $96.4 \%, 86.6 \%$ and $74.8 \%$, respectively (9). The median age at HCC diagnosis in these HIV/HCV co-infected 14 cases of Child-Pugh A was similarly young, 49 years.

RFA is generally considered to be, on the one hand, less invasive than surgical resection, with less bleeding, and on the other, better than TACE at controlling small HCCs (10). The advantage of RFA over hepatectomy for HCC with lesion size and number less than or equal to $3 \mathrm{~cm}$ and 3 , has not yet been elucidated, and the JSH guideline equally recommends both for Child-Pugh A cases; however, hepatectomy is typically regarded as the first choice for small/solitary HCC (4). Accordingly, we defined the 4 cases which received RFA as the primary treatment for small/ solitary HCC as "JSH guideline unmet". However, even if these 4 cases had been defined as "JSH guideline met", still only $65 \%$ (11/17) would have received the treatment recommended by the JSH guideline. Actually, in the JSH guideline, "liver damage grade" should be considered 
rather than Child-Pugh grade, but no cases underwent indocyanine green test in this study, so we could not evaluate liver damage grade. However, we believe that most of the Child-Pugh A cases had liver damage grade A, so this issue did not affect the results of this study.

The discrepancy of the chosen treatment might reflect not only the bleeding risk, but also the degree of involvement of the hepatologist or liver surgeon in charge of the patients. Because HIV/HCV coinfected patients are typically treated by a multi-disciplinary team, the hepatologist/liver surgeon might be less profoundly involved in these cases. In fact, most of the data in this study were from centers in which the infectious disease doctors or hematologists were the patients' attending physicians. Certainly, multidisciplinary collaboration is established in all centers participating in this study, but issues of professional expertise might pose challenges for the physicians most closely involved in the case in terms of choosing HCC treatment. And honestly, we are concerned that most centers have few interests in this important issue, because of far less response to our first question to participate in this study $(139 / 444,31 \%)$. We should try to enlighten physicians who especially are responsible for HIV and/or hemophilia.

Also, the performance status of the patients might affect the treatment choice in this study, because daily activity is generally disturbed in hemophilic patients, mainly due to hemophilic arthropathy (11). Because the actual performance status at HCC diagnosis was not recorded in this study, we analyzed the data assuming the patients had good performance status. Generally, performance status should be considered when it can affect the general condition after the treatment, as in elderly patients or those with multiorgan complications. Accordingly, we believe that standard treatment should be considered for patients in this study even with disturbed performance status, because the patients were generally young without significant organ complications.

Not only HCC treatment, but HCV treatment might pose challenges to standardization because of several concerns such as the interaction between anti-HIV treatment and DAA for HCV (12). Only 3 cases in this study had received DAA before the diagnosis of HCC, all of which could achieve SVR. The safety and efficacy of DAA in HIV/HCV coinfection has recently been confirmed, so that DAA might contribute to the improvement of both HCV/ HCC treatment in this population, as has already been shown in Western countries $(13,14)$. As mentioned above, the reason of small number in HCV treatment with DAA in Japan might be the reluctant to adopt it because of the possible insufficient collaboration between the hepatologists and hematologists, and/or HIV physicians. The importance of cooperation should be emphasized again, and HIV/HCV coinfected patients might be better to receive DAA with the priority hereafter.

Interestingly, among 9 cases of HCV-RNA negative at the diagnosis of HCC, $3(33 \%)$ were considered to have naturally eradicated the virus because they had no history of $\mathrm{HCV}$ treatment. This incidence of spontaneous clearance of $\mathrm{HCV}$ is as high as the data reported by Westbrook et al. (15) as $18 \%$ to $34 \%$ in general HCVpositive population.

Naturally, early detection of HCC is extremely important especially in hemophilic patients because of limited treatment option, but for the patients with liver failure, LT can be the treatment of choice. In HIV/HCV coinfected patients, the indication and timing of LT have been considered difficult to assess, because even in Child-Pugh A cases, the patient suddenly can deteriorate with the onset of variceal bleeding or hepatic coma, due to noncirrhotic portal hypertension $(16,17)$. Also, postoperative management is complicated by the interaction between ART and immunosuppression (18). Currently, the prognosis of LT for HCC in cases with HIV/HCV coinfection has significantly improved, considering the timing of LT and treatment for both HIV and HCV, especially the development of ART and DAA (19). Accordingly, LT should be considered not only for Child-Pugh C patients, but also for Child-Pugh B cases when the HCC lesions are small in size and number.

There are several limitations in this study, including its being a retrospective analysis for a small number of cases without statistical comparison analysis, and the reference of results to the current treatment guideline regardless of the year of diagnosis. Also, the diagnosis of HCC might be not accurate and should be strictly defined according to the unified protocol, but we believe the accuracy of the imaging diagnosis of HCC without pathological specimen in each institution should be acceptable, because all of which has radiology specialists. Although there is no actual national protocol of the HCC diagnosis in Japan, the typical imaging findings of ultrasound, CT, and MRI are shown in treatment guideline in Japan (4). Another limitation was the bias in gender, because of all of the cases in this study was male. Generally, the population of HCV-related HCC is more in men, but it might be difficult to discuss the impact of gender on the outcome of HCC in HIV/HCV 
coinfection, because there are no female patients in current study. However, in spite of these limitations, we believe that the information of this paper may be helpful to physicians in charge of treating HIV/HCV coinfected patients, because the treatment modality for HCC has not changed significantly during these years, especially for HCC lesions that are small in size and number.

In conclusion, Japanese hemophilic HCC patients with $\mathrm{HIV} / \mathrm{HCV}$ coinfection may not routinely receive standard treatment due to their bleeding tendency and several complications related to HIV/HCV coinfection. Strong multi-professional collaboration including a hepatologist/ liver surgeon as well as hematologist and infectious disease specialist could be the key to rectifying this complicated situation.

\section{Acknowledgments}

Funding: This study was partially supported by a Health and Labor Sciences Research Grant from the Ministry of Health, Labor and Welfare Japan, regarding Research on Liver Transplantation for HIV/HCV coinfected Patients ("Eguchi project").

\section{Footnote}

Reporting Checklist: The authors have completed the STROBE reporting checklist. Available at https://dx.doi. org/10.21037/jgo-21-157

Data Sharing Statement: Available at https://dx.doi. org/10.21037/jgo-21-157

Peer Review File: Available at https://dx.doi.org/10.21037/ jgo-21-157

Conflicts of Interest: All authors have completed the ICMJE uniform disclosure form (available at https://dx.doi. org/10.21037/jgo-21-157). The authors have no conflicts of interest to declare.

Etbical Statement: The authors are accountable for all aspects of the work in ensuring that questions related to the accuracy or integrity of any part of the work are appropriately investigated and resolved. The study approved first by the institutional review board of Nagasaki University Hospital (approval No. 16062709), and then by the review boards of all participating institutions. In principle, written informed consent was not necessary for retrospective and observational study depending on each institutional regulation, but instead of it, the contents of the study were informed on website in Japanese (https://www. med.nagasaki-u.ac.jp/surgery2/hlw_kaken/index.html). The study was conducted in accordance with the Declaration of Helsinki (as revised in 2013).

Open Access Statement: This is an Open Access article distributed in accordance with the Creative Commons Attribution-NonCommercial-NoDerivs 4.0 International License (CC BY-NC-ND 4.0), which permits the noncommercial replication and distribution of the article with the strict proviso that no changes or edits are made and the original work is properly cited (including links to both the formal publication through the relevant DOI and the license). See: https://creativecommons.org/licenses/by-nc-nd/4.0/.

\section{References}

1. Koike K, Tsukada K, Yotsuyanagi H, et al. Prevalence of coinfection with human immunodeficiency virus and hepatitis C virus in Japan. Hepatol Res 2007;37:2-5.

2. Yotsuyanagi H, Kikuchi Y, Tsukada K, et al. Chronic hepatitis $\mathrm{C}$ in patients co-infected with human immunodeficiency virus in Japan: a retrospective multicenter analysis. Hepatol Res 2009;39:657-63.

3. European Association for the Study of the Liver. EASL Clinical Practice Guidelines: Management of hepatocellular carcinoma. J Hepatol 2018;69:182-236. Erratum in: J Hepatol 2019;70:817.

4. The Japan Society of Hepatology. JSH HCC Guidelines 2017. Available online: https://www.jsh.or.jp/medical/ guidelines/jsh_guidlines/medical/examination_jp_2017. html

5. Al-Khazraji A, Patel I, Saleh M, et al. Identifying Barriers to the Treatment of Chronic Hepatitis C Infection. Dig Dis 2020;38:46-52.

6. Cacoub P, Rosenthal E, Halfon P, et al. Treatment of hepatitis $\mathrm{C}$ virus and human immunodeficiency virus coinfection: from large trials to real life. J Viral Hepat 2006;13:678-82.

7. Eguchi S, Takatsuki M, Soyama A, et al. The First Case of Deceased Donor Liver Transplantation for a Patient with End-Stage Liver Cirrhosis Due to Human Immunodeficiency Virus and Hepatitis C Virus Coinfection in Japan. Jpn J Infect Dis 2016;69:80-2.

8. Tsukada K, Sugawara Y, Kaneko J, et al. Living donor 
liver transplantations in HIV- and hepatitis C viruscoinfected hemophiliacs: experience in a single center. Transplantation 2011;91:1261-4.

9. The Liver Cancer Study Group of Japan. The 21th Japanese Registry for Primary Liver Cancer. Available online: https://doi.org/10.2957/kanzo.61.645

10. Dong W, Zhang T, Wang ZG, et al. Clinical outcome of small hepatocellular carcinoma after different treatments: a meta-analysis. World J Gastroenterol 2014;20:10174-82.

11. Goddard NJ, Mann HA, Lee CA. Total knee replacement in patients with end-stage haemophilic arthropathy: 25year results. J Bone Joint Surg Br 2010;92:1085-9.

12. Poizot-Martin I, Naqvi A, Obry-Roguet V, et al. Potential for Drug-Drug Interactions between Antiretrovirals and HCV Direct Acting Antivirals in a Large Cohort of HIV/ HCV Coinfected Patients. PLoS One 2015;10:e0141164.

13. Machado SM, Vigani AG, Leite AG, et al. Effectiveness of direct-acting antivirals for hepatitis $\mathrm{C}$ virus infection in hepatitis C/HIV coinfected individuals: A multicenter study. Medicine (Baltimore) 2020;99:e21270.

14. Corma-Gómez A, Macías J, Lacalle-Remigio JR, et al.

Cite this article as: Takatsuki $M$, Natsuda $K$, Hidaka $M$, Sawada K, Shindo M, Endo T, Hagiwara T, Yotsuyanagi H, Koibuchi T, Tsukada K, Uemura H, Hayashi K, Uehira T, Mita E, Yamamoto M, Takahama S, Eguchi S. The treatment choices and outcome of hepatocellular carcinoma in hemophilic patients with human immunodeficiency virus/hepatitis $\mathrm{C}$ virus (HIV/HCV) coinfection due to contaminated blood products in Japan. J Gastrointest Oncol 2021;12(6):2952-2959. doi: 10.21037/ jgo-21-157
Human Immunodeficiency Virus (HIV) Infection Is Associated With Lower Risk of Hepatocellular Carcinoma After Sustained Virological Response to Direct-acting Antivirals in Hepatitis C Infected Patients With Advanced Fibrosis. Clin Infect Dis 2021;73:e2109-16.

15. Westbrook RH, Dusheiko G. Natural history of hepatitis C. J Hepatol 2014;61:S58-68.

16. Sulkowski MS, Mehta SH, Torbenson MS, et al. Rapid fibrosis progression among HIV/hepatitis C virus-coinfected adults. AIDS 2007;21:2209-16.

17. Martín-Carbonero L, Tuma P, Vispo E, et al. Treatment of chronic hepatitis C in HIV-infected patients with compensated liver cirrhosis. J Viral Hepat 2011;18:542-8.

18. Schvarcz R, Rudbeck G, Söderdahl G, et al. Interaction between nelfinavir and tacrolimus after orthoptic liver transplantation in a patient coinfected with HIV and hepatitis C virus (HCV). Transplantation 2000;69:2194-5.

19. Agüero F, Forner A, Manzardo C, et al. Human immunodeficiency virus infection does not worsen prognosis of liver transplantation for hepatocellular carcinoma. Hepatology 2016;63:488-98. 